\title{
A Randomized Trial of Low-Cost Mesh in Groin Hernia Repair
}

Jenny Löfgren et al.

N Engl J Med 2016; 374:146-153 January 14, 2016DOI: 10.1056/NEJMoa1505126

A double-blind, randomized, controlled trial, comparing a low-cost mesh with a commercial mesh (both lightweight) for repairing a groin hernia in adult men in eastern Uganda who had primary, unilateral, reducible groin hernias, was performed by four qualified surgeons. A total of 302 patients were included in the study, and the follow-up rate was $97.3 \%$ after 2 weeks and $95.6 \%$ after 1 year. Hernia recurred in one patient $(0.7 \%)$ assigned to the low-cost mesh and in no patients assigned to the commercial mesh. However, postoperative complications occurred in 44 patients (30.8\%) assigned to the low-cost mesh and in 44 patients (29.7\%) assigned to the commercial mesh. In conclusion, rates of hernia recurrence and postoperative complications did not differ significantly between men undergoing hernia repair with low-cost mesh and those undergoing hernia repair with commercial mesh.

\section{The Effect of Workers' Compensation Status on Outcomes of Cervical Disk Arthroplasty A Prospective, Comparative, Observational Study}

\section{Matthew F. Gornet et al.}

J Bone Joint Surg Am, 2016 Jan 20; 98 (2): 93 -99 . http://dx.doi.org/10.2106/JBJS.0.00324

The aim of this study was to compare the outcomes of cervical disk arthroplasty between patients receiving and those not receiving Workers' Compensation. The Authors analyzed the patient-reported outcomes, reoperations, complications, and return-to-work status 1 year after surgery in an observational cohort of consecutive patients who underwent single-level or multilevel cervical disk arthroplasty for symptomatic cervical disk conditions. Of the 189 patients who underwent cervical disk arthroplasty, 144 received Workers' Compensation and 45 did not. The mean scores of all patient-reported measures improved significantly from preoperative baseline to 1 year after surgery. However, the improvement in patient-reported outcomes did not differ significantly between the Workers' Compensation and the non-Workers' Compensation groups.

No significant difference was observed in the rate of reoperations (7.6\% for those receiving Workers' Compensation compared with $13.3 \%$ for those not receiving Workers' Compensation), and complications (2.8\% compared with 4.4\%, respectively) between the two groups. Although the patients receiving Workers' Compensation had significantly more days off before returning to work, the proportion of patients who had returned to work was comparable at 1 year after surgery.

In conclusion, after cervical disk arthroplasty, the outcomes of patients receiving Workers' Compensation were similar to those of patients not receiving Workers' Compensation in terms of patient-reported outcomes, surgery-related complications, reoperations, and return-to-work status. Finally, patients receiving Workers' Compensation remained off work for a longer interval than did patients not receiving Workers' Compensation.

\footnotetext{
* This update summarizes selected articles that were published in 2016 in distinguished medical scientific journals.

** Prepared by:

Şinasi ÖZSOYLU, MD e-mail: sinasiozsoylu@hotmail.com M. Nedim Aytekin, MD e-mail: nedimaytekin@hotmail.com
} 


\section{Intensified Antituberculosis Therapy in Adults with Tuberculous Meningitis}

\section{A. Dorothee Heemskerk et al.}

N Engl J Med 2016; 374:124-134 January 14, 2016 DOI: 10.1056/NEJMoa1507062

Tuberculosis meningitis is one of the most severe infections of the central nervous system. The best antimicrobial regimen for tuberculosis meningitis includes isoniazid, rifampin, pyrazinamide, and streptomycin. Despite most of these antimicrobial agents are bactericidal, optimum cerebrospinal fluid levels can be obtained when the meninges are inflamed.

Several studies are available in the literature regarding the optimal dose of rifampin in tuberculosis meningitis. Heemskerk et al evaluated standard antituberculosis treatment versus intensified treatment that included additional $5 \mathrm{mg} /(\mathrm{kg}$-day) rifampin and $20 \mathrm{mg} /(\mathrm{kg}$-day) levofloxacin in tuberculosis meningitis.

This randomized, double-blinded, placebo controlled study from Vietnam among 817 HIV-infected and non-infected adult patients revealed that the mortality rate is similar between the standard treatment group and the intensified treatment group. Severe neurological disability at treatment initiation, HIV infection, and drug-resistance were found to be predictors of mortality. Among patients who were infected with HIV, high level of CD cell count was related to reduced mortality. They found no statistically significant difference between both groups in terms of neurological complications. They also reported that an additional dose of rifampin and levofloxacin has no effect on adverse events causing treatment interruptions.

Results of this study are important because the study included high number of patients, carefully designed, and well randomized. Unfortunately, authors did not find any effect of intensified regimen on reducing the mortality rate.

\section{Swimming Improves Pain and Functional Capacity of Patients with Fibromyalgia: A Randomized Controlled Trial}

Fernandes G. et al.

Arch Phys Med Rehabil. 2016 Feb 19. pii: S0003-9993(16)00101-5. doi: 10.1016/j.apmr.2016.01.026

The aim of this study was to explore the effect of swimming on pain, functional capacity, aerobic capacity, and quality of life on patients with fibromyalgia.

This randomized controlled study included 75 female patients (age ranged between 18 and 60 years) with fibromyalgia randomly categorized into a swimming group $(n=39)$ and a walking group $(n=36)$. The swimming group performed 50 minutes of swimming three times a week for 12 weeks, with a heart rate of 11 beats under the anaerobic threshold, while the walking group performed walking with the heart rate under the anaerobic threshold, with the same duration and frequency of the swimming group. 
Participants were assessed before and after (at 6 weeks and at 12 weeks) the exercise protocols. The main outcome measure was the visual analog scale for pain. The other measurements were: Fibromyalgia Impact Questionnaire and SF-36 questionnaire for quality of life; spiroergometric test for cardiorespiratory variables; and Timed Up-and-Go Test for functional performance.

In both groups, pain was improved after the 12-week program, but no difference was observed between the groups ( $p=$ 0.658). The similar results were found regarding functional capacity and quality of life. Additionally, the aerobic capacity did not change significantly in either groups.

These results have shown that swimming, like walking, is an effective method for reducing pain and improving both functional capacity and quality of life in subjects with fibromyalgia.

\section{A Phase III Clinical Trial Showing Limited Efficacy of Autologous Mesenchymal Stem Cell Therapy for Spinal Cord Injury}

Oh SH. et al.

Neurosurgery. 2016 Mar;78(3):436-47

Oh and all presented the effect of mesenchymal stem cells (MSCS) on American Spical Injury Association B status patients who had more than 12 months of cervical injury, and no neurological changes during the recent 3 months vigorous rehabilitation. Autologous MSCs were injected into the intramedullary area and subdural space of these patients. The authors evaluated the results after 6 months.

Of 16 patients, 2 showed improvement in the neurological status. These patients with neurological improvement showed the appearance of continuity in the spinal tract by diffusion tensor imaging. The authors did not observe adverse effects associated with the MSC injection.

According to the authors, single MSC injection into the intramedullary and intradural spaces is safe but has a very weak thearapeutic effect compared with multiple MSC injection. Authors recommend further clinical trials for analyzing the effect of MSCS. 